\title{
Inter/transdisciplinariedad y universidad: patrones de desplazamiento e interactividad disciplinar
}

Interdisciplinary/Transdisciplinary and the University:

Patterns of Disciplinary Mobility and Interactivity

Inter/transdisciplinarité et université: patrons de déplacement et interactivité discipliner

Inter/transdisciplinaridade e universidade:

padrões de deslocamento e de interatividade disciplinar

Fecha de recepción: 1 DE ABRIL DE 2013/ Fecha de aceptación: 5 DE OCTUBRE DE 2013

Encuentre este artículo en http://magisinvestigacioneducacion.javeriana.edu.co/

doi:10.11144/Javeriana.M6-13.ITUP

ESCRITO POR IVÁN OlIVA-FIgUeroa UnIVERSIDAD AUSTRAL

Sector Isla Teja, Los Ríos, Chile ivanoliva@uach.cl

\section{Resumen}

El artículo problematiza las disyunciones epistémicas entre los diversos campos disciplinares en el contexto universitario, como consecuencia de flexibilidad limitada, sobreespecialización del currículo y sistemas de conocimiento disciplinares autorreferenciados.

Se levantaron indicadores de desplazamiento e interactividad disciplinar estudiantil, lo que permitió generar una óptica parcial de discusión en torno al fenómeno de la inter/transdisciplinariedad en espacios universitarios. En términos globales, fue posible evidenciar una significativa rigidez y clausura operativa del currículo flexible, lo que configura un nodo crítico que condiciona parcialmente la emergencia de matrices comunicativas más densas y de esquemas de docencia con mayores grados de inter/transdisciplinaridad.

\section{Palabras clave autor}

Universidad, transdisciplinariedad, flexibilidad curricular.

\section{Palabras clave descriptor}

Aproximación interdisciplinaria en educación, universidadesChile, desarrollo curricular.
TOMÁs KOCH-EWERTZ Universidad de Playa Ancha de Ciencias de la Educación Valparaíso, Chile tomas.koch@upla.cl

JOHn QUINTERO-TAPIA Universidad SAN Sebastí́n VALDIVIA, CHILE john.quintero@uss.cl

Para citar este artículo / To cite this article / Pour citer cet article / Para citar este artigo

Oliva-Figueroa, I., Koch-Ewertz, T., \& Quintero-Tapia, J. (2014). Inter/transdisciplinariedad y universidad: patrones de desplazamiento e interactividad disciplinar. magis, Revista Internacional de Investigación en Educación, 6 (13), 99-112.

\section{Transferencia a la práctica}

La discusión e información presentada en este trabajo pretende contribuir al fomento de procesos formativos con mayores grados de flexibilidad y multirreferencialidad disciplinar en el contexto de las universidades contemporáneas. 
Key words author

University, Transdisciplinarity,

Flexible Curriculum.

\section{Key words plus}

Interdisciplinary Approach in Education, Universities and Colleges-Chile, Curriculum Development.

\section{Abstract}

This paper focuses on the problem of epistemic disjunctions between the different disciplinary fields within the context of universities, as a consequence of limits to flexibility, excessive curricular specialisms and systems of self-referenced disciplinary knowledge. The study included the construction of indicators of disciplinary mobility and interactivity of students, which allowed us to discuss interdisciplinarity and transdisciplinarity at the university. In general terms, the outcome of the study revealed the significant rigidity and practical restrictions of flexible curriculum, conditions that limit the emergence of denser communication schemes and more interdisciplinarity and transdisciplinarity in higher education.

\section{Transference to practice}

This paper includes information and discussions that seeks to contribute to the encouragement of more flexible and multidisciplinary referenced education processes, within the context of contemporary universities.

\section{Mots clés auteur}

Université, transdisciplinarité, flexibilité curriculaire.

\section{Mots clés descripteur}

Interdisciplinarité dans l'enseignement, universités-Chili, Programmes d'études-Planification.

\section{Résumé}

L'article problématise, dans le contexte universitaire, les disjonctions épistémiques entre les divers champs disciplinaires, en tant que conséquence d'une flexibilité limité, par rapport à la spécialité du curriculum et les systèmes de connaissance disciplinaires autoréférences. On a relevé des indicateurs de déplacement et interactivité discipliner des étudiants, cela a permis donner une optique partielle du débat par rapport au phénomène de l'inter/transdisciplinarité dans les espaces universitaires. En termes globaux, il a été possible de montrer, une rigueur significative et fermeture opérative du curriculum flexible, ce qui façonne un nœud critique qui conditionne partiellement, l'émergence de matrices communicatives plus denses et de schémas d'enseignement avec majeures degrés d'inter/ transdisciplinarité.

\section{Transfert à la pratique}

Le débat et information présenté dans ce travail vise contribuer au développement de processus formatifs avec majeure degré de flexibilité et multiréférentialité discipliner dans le contexte des universités contemporaines.

\section{Palavras-chave autor}

Universidade, transdisciplinaridade, flexibilidade curricular.

\section{Palavras-chave descritor} Abordagem interdisciplinar do conhecimento na educação, universidades e faculdades-Chile, desenvolvimento curricular.

\section{Resumo}

O artigo problematiza, no contexto universitário, as disjunções epistêmicas entre os diversos campos disciplinares, como consequência de uma flexibilidade limitada, de uma sobre-especialização do currículo e de sistemas de conhecimento disciplinares autorreferenciados.

Levantaram-se indicadores de deslocamento e de interatividade disciplinar estudantil, o que permitiu gerar uma visão parcial da discussão em torno do fenômeno da inter/ transdisciplinaridade em espaços universitários. Em termos globais, foi possível evidenciar uma significativa rigidez e um fechamento operativo do currículo flexível, o que configura um ponto crítico que condiciona parcialmente a emergência de matrizes comunicativas mais densas e de esquemas de docência com maiores graus de inter/transdisciplinaridade.

\section{Transferência à prática}

A discussão e informação apresentadas neste trabalho pretendem contribuir no fomento de processos formativos com maiores graus de flexibilidade e multirreferencialidade disciplinar no contexto das universidades contemporâneas. 


\section{Introducción}

La discusión multidimensional en torno a la congruencia, coherencia y pertinencia de las universidades en el escenario global es un campo inaprensible para cualquier programa particular de investigación. Las perspectivas de desarrollo de una de las instituciones más reconocidas e importantes de la sociedad contemporánea se mueven en diversos e imbricados niveles de interpretación que atraviesan dominios estructurales y no estructurales del sistema. Una aproximación interpretativa compleja a la educación terciaria es una tarea donde confluyen los planos epistemológicos, sociológicos, antropológicos, políticos y económicos del fenómeno $y$, en estos términos, resiste protocolos de investigación reduccionistas, deterministas y unidimensionales.

Los actuales desafíos de la educación superior en Chile y el mundo no responden a lógicas triviales, sino que exceden consideraciones cuantitativas, estructurales y de proyección lineal. Por tanto, los desafíos se sitúan en puntos de inflexión cualitativos que contemplan los procesos complejos de "vigilancia epistemológica" en torno a la pertinencia de los patrones de comunicación y la organización del conocimiento que generan y proyectan.

Esto conlleva la necesidad de desarrollar nuevas formas de conocimiento, en un tránsito de orden epistemológico-paradigmático transversal a todos los dominios de conocimiento. Una prospectiva hacia la reintegración de saberes dispersos en distintas disciplinas universitarias, con un énfasis no solo en la acumulación, organización y comunicación inter y transdisciplinaria (Oliva, 2008). No obstante, como advierte Nicolescu (1996), es necesaria una discusión seria y constructiva, dado que:

[...] el desarrollo acelerado de la perspectiva transdisciplinaria naturalmente se ha acompañado del peligro de múltiples desviaciones: la desviación mercantil, la desviación de la búsqueda de nuevos modos de dominación del otro, cuando no simplemente la tentativa de vaciar la nada en el vacío por la adopción de un eslogan gracioso carente de todo contenido (p. 3).

En este contexto, las nociones de organización y comunicación no son triviales, puesto que significan asumir una perspectiva de investigación orientada a los patrones de comunicación, significación y pertinencia asociados con los conocimientos proyectados por los sistemas de educación terciaria. Desde esta base, la organización del conocer humano se transforma en una tarea capital, aleatoria e incierta; para ello, hay que comprender que la revolución se presenta hoy no en la oposición entre ideas erradas y verdaderas, sino en el terreno de la complejidad del modo de organización de dichas ideas, lo que requiere pensar lo educativo de forma compleja (Morin, 1992). Cuanto más progresa la crisis, más progresa la incapacidad para pensarla; cuantas más investigaciones se desarrollan en torno a las diversas problemáticas contemporáneas, económicas, ecológicas y políticas globales, más se comprende la necesidad de enfoques complejos y transdisciplinarios distantes de programas especializados, aislados y monodisciplinares (Rosnay, 1977; Berman, 1981; Nemeth-Baumgartner 1993; Capra, 2002; Morin, 1999; 2006; 2008; Hirsch Hadorn et al., 2008; Leavy, 2011).

Desde esta convergencia, la problemática se torna organizacional, en una dimensión no solo estructural, alcanzado el registro de profundos procesos epistemológicos latentes a los esquemas manifiestos de las formas de interacción y comunicación disciplinaria predominante en las
Descripción del artículo | Article description | Description de l'article | Artigo descrição

Este artículo de investigación muestra resultados dentro del contexto de un programa de investigación y desarrollo en complejidad, transdisciplina y universidad. El desarrollo teórico, metodológico y los resultados parciales presentados forman parte de los proyectos Fondecyt 11080193 y 1120863. 
universidades contemporáneas. Derivado de lo anterior, sostenemos que un importante nodo crítico y la deficiencia estructural de los modelos curriculares y la política de educación terciaria están asociados con la capacidad de las universidades para organizar y comunicar campos disciplinares aislados en un orden más interactivo y complejo.

En el amplio registro de esta problemática, el objetivo transversal del programa de investigación fue la comprensión de los sustratos epistemológicos, curriculares y organizacionales que pudiesen estar en la base de la excesiva autorreferencia y disociación entre diversos campos de desarrollo científico.

\section{La disyunción disciplinaria como problemática organizativa y epistémica}

La noción de disciplina puede ser definida como una categoría organizadora dentro del conocimiento científico, en el que se instituyen la división y la especialización. Por más que se inserte en un contexto científico amplio, una disciplina tiende a la autonomía por medio de la delimitación de sus fronteras técnicas, metodológicas y epistemológicas (Morin, 1999). En estos términos, el fenómeno de la organización inter/ transdisciplinaria está asociado histórica y epistemológicamente con la disciplinariedad científica (Lenoir, Hasni \& Larose, 2007). No se puede quebrar aquello que ha sido creado por las disciplinas; no se puede quebrar todo encierro. La problemática de la organización disciplinar, como el problema de la vida, requiere que una disciplina sea a la vez abierta y cerrada (Morin, 1992).

La organización disciplinaria tiene un correlato en la génesis de las universidades modernas en el siglo XIX (Morin, 1999; Bunge, 2004), aunque es posible rastrear sus orígenes en la Grecia antigua, en los esfuerzos por establecer límites y relaciones entre los diversos saberes en mapas de conocimiento occidental (Miñana, 2002). En este sentido, el devenir de las disciplinas se inscribe en la historia de la sociedad; por ello, la organización disciplinar puede ser abordada desde la sociología de las ciencias (Kuhn, 1962; Bunge, 1991; 2004). Además, posee una dimensión epistemológica y comunicacional abocada al entendimiento de las formas de organización del conocimiento disciplinar y sus procesos de clausura y apertura (Morin, 1999).

En este escenario, la universidad es un espacio neurálgico en donde confluyen diversas matrices disciplinares, definidas en gran medida como un correlato de especialización, lenguaje autorreferenciado y experticia temática. Con ello se arriesga el debilitamiento de las interrelaciones, el diálogo y la confluencia transdisciplinar en la comprensión holística de la con- tinuidad, especificidad y coproducción de los diversos niveles de organización de los fenómenos y las problemáticas contemporáneas.

En síntesis, la cuestión en torno a los procesos de fractura epistemológica entre los dominios emerge desde una compleja matriz de identidades disciplinares que arriesgan clausuras comunicacionales muy perjudiciales para el desarrollo multidimensional de la educación terciaria en Chile y el mundo. En este contexto, Flórez y Millán (2002) postulan que, mientras los estudiantes de distintos contextos formativos se distancian de currículos cerrados y especializados, el mercado laboral envía señales ambiguas, genera una demanda que no se reduce a especialistas disciplinares y requiriere profesionales con una formación cada vez más inter/transdiciplinar, aún más fuerte que la disciplinar. Derivado de ello, el problema de las disciplinas como referentes adecuados para la organización de las universidades se presenta como uno de los puntos de ruptura práxica más importantes del siglo XXI.

\section{Organización disciplinar: una revisión del estado del arte}

Los procesos organizacionales de interactividad entre dominios disciplinares responden a una amplia variedad de tipologías y denotaciones, entre las que se hallan las nociones de multi, pluri, inter, trans y metadisciplinariedad. Los derroteros epistemológicos que les ofician de base han sido tratados desde la epistemología (Piaget, 1966; Morin, 1998; 2006; Nicolescu, 1996; Motta, 2000; Reynoso, 2009), la filosofía y la sociología de la ciencia (Kuhn, 1962; Lakatos \& Musgrave 1970; Feyerabend, 1975; Bunge, 2004), la metodología de la investigación (Greckhamer, Kro-Ljungberg, Cilesiz \& Hayes, 2008), la lingüística (Parodi, 2008), la morfología de redes sociales y las mediaciones tecnológicas (Reynoso, 2011; Gherab, 2009) y, en particular las Ciencias de la Educación (Miñana, 2002; Oliva, 2008, 2010). Esta interactividad disciplinar en el sistema universitario chileno ha sido investigada desde enfoques basados en la gestión y política universitaria (Salazar, 2003; Cáceres \& Cristi, 2003), desde los sustratos epistemológicos (Oliva, 2008; Díaz, 2010; Soler, 2011; Silva 2011) y desde procesos de cooperación en investigación (Villarroel \& Firmani 2002; Cárdenas et al., 2012).

En el ámbito de la gestión universitaria, se han detectado condicionantes significativas para el desarrollo de procesos transversales, cuyo resultado depende de la contribución de más de una unidad académica (Cáceres, 2007). En el contexto de las lógicas de investigación, Villarroel y Firmani (2002) evidenciaron la baja interactividad disciplinar de los equipos de 
investigación en torno a problemáticas de relación compleja sociedad humana-ambiente'

En el escenario internacional, desde cánones cualitativos de investigación, Jeffrey (2003) acentúa que, si bien sus resultados no son generalizables, los puntos de unificación de diversos campos disciplinares no son solo una alianza pragmática que funciona de manera mecánica. Austin, Park y Goble (2008) han descrito que los modelos multi y transdisciplinarios presentan profundas condicionantes de orden epistémico que no han sido comprendidas con suficiencia. En el área de las Ciencias de la Educación, Lenoir y otros autores (2007) han estudiado los enfoques conceptuales de profesores en formación y en ejercicio, en relación con el trabajo interdisciplinario, con evidencia de bases epistemológicas superficiales y una significativa estabilidad conceptual en torno a la jerarquización disciplinar, lo que implicaría condicionantes epistemológicas en lo referido a mayores grados de interactividad disciplinar en el proceso educativo.

\section{Grados de autonomía y flexibilidad curricular: sustratos para la emergencia de esquemas inter/transdisciplinarios}

Sostenemos que la flexibilidad curricular es una condición básica para el desarrollo de mayor interactividad entre estudiantes y académicos agenciados a diversos campos disciplinares. No obstante, es necesario precisar que, en este ámbito de reflexión, la noción de flexibilidad curricular es entendida como un sustrato basal y un referente particular en el entendimiento multidimensional de procesos inter/transdisciplinarios en contextos universitarios.

En este registro, los procesos de desplazamiento e interactividad disciplinar promovidos en contextos de flexibilidad curricular posibilitan la instauración de redes cognitivas densamente relacionadas, que dan forma, en el sentido de Kelly (1995), a una morfología adaptada para una compleja interacción creciente y para pautas de desarrollo impredecibles que surgen del poder creativo de esta interacción.

De manera simultánea, la flexibilidad, definida como una relación de grados de plasticidad, contingencia y posibilidades de reconfiguración, conlleva el reconocimiento de los procesos de autonomía del aprendizaje. En el ámbito amplio de las epistemologías constructivistas, situamos la noción de autonomía con

1 Se estudiaron los equipos disciplinares de 65 investigaciones sustentadas en problemáticas de interacción sociedad humana-ambiente, lo que supone la necesidad epistemológica y metodológica de programas inter y transdisciplinarios. Esto evidencia que solo un $29 \%$ incluía investigadores de más de una disciplina y solo un $6 \%$ convocaba a investigadores tanto de las ciencias naturales como de las ciencias sociales. referencia al énfasis en los procesos auto(eco)organizativos inherentes a todo acto cognitivo, lo que supone un estado de conciencia en torno a la imposibilidad de instruir o determinar el curso cognitivo de un sistema autónomo y, con ello, restringir sus posibilidades de contingencia, relación o flexibilidad.

De este modo, la significancia, la pertinencia y la operatividad de la flexibilidad curricular está posibilitada y condicionada por el reconocimiento de las dimensiones de autonomía asociadas con los procesos de aprendizaje. En última instancia, los grados de flexibilidad curricular intencionados en los procesos formativos traen un reconocimiento implícito de la autonomía de todo acto cognitivo y conforman una dinámica recursiva entre flexibilidad y autonomía.

Dentro del marco de especificidad de la educación superior en Chile y a partir de estándares internacionales, el informe OCDE (2009) ha señalado la flexibilidad limitada y la sobreespecialización del currículo como deficiencias estructurales genéricas del sistema. En relación con la flexibilidad limitada, desde la revisión curricular de una variedad de programas académicos e instituciones se indica el énfasis de un currículo rígido, con opciones muy limitadas o sin electivas, una vez que el estudiante escoge un área de especialización. Esta flexibilidad limitada, además, es uno de los principales impedimentos para la articulación y movilidad entre programas y niveles académicos en Chile, en cuanto a la convergencia curricular entre los niveles técnico superior, profesional y universitario (Gaete \& Morales, 2011).

En lo referido a la sobreespecialización, el mismo informe OCDE (2009) afirma que la mayor parte de los programas académicos remarca la preparación para un campo de estudio específico y enfatiza en una variedad de materias orientadas profesionalmente, pero no incluyen cursos de educación general, lo que muestra que los estudiantes deben elegir un campo académico al inicio de sus estudios. El informe asevera también que, salvo pocas excepciones, no se permite la movilidad lateral entre programas académicos, incluso dentro de la misma institución. En algunos casos, se ha incorporado cierta disponibilidad de cursos optativos dentro del mismo campo de estudio. Este factor, combinado con la limitada orientación vocacional en la enseñanza secundaria, tiene gran influencia en las tasas de deserción ${ }^{2}$ en la educación terciaria en el país.

A modo de síntesis y desde la base de lo documentado por la Unesco en torno a educación superior, González (2011) establece que el paradigma del

2 El desarrollo de programas remediales y de nivelación, así como una discusión referida a las causas de la deserción universitaria en Chile son discutidos en detalle en el Informe final del estudio sobre causas de la deserción universitaria (Centro Micro Datos, 2008). 
aprendizaje contemporáneo supone, entre otras dimensiones, la redefinición hacia un currículo más flexible, sistémico y reflexivo, proyectado desde la base de nuevos territorios temáticos con la incorporación de enfoques multirreferenciales, del diálogo de saberes, de metodologías y de la articulación de las ciencias, las humanidades y las artes, para posibilitar la movilidad vertical y horizontal de estudiantes y profesores.

\section{Metodología}

Las estrategias metodológicas integraron enfoques cuantitativos y cualitativos, tanto en triangulación como en generación, análisis e interpretación de información, bajo un diseño general quan a qual (cuantitativo a cualitativo).

El programa global de investigación se orientó a la cartografía de diversos nodos críticos (estructurales, curriculares y epistemológicos) inherentes al desarrollo significativo, sustentable y pertinente del esquema de trabajo inter/transdisciplinario en el entorno universitario chileno.

Los criterios de inclusión consideraron tres universidades chilenas con las siguientes características ${ }^{3}$ : instituciones tradicionales y de vocación pública, definidas en sus directrices políticas como universidades complejas (desarrollo de docencia, investigación y extensión), formación de pregrado y posgrado, acreditación institucional ante la CNA ${ }^{4}$ y con una organización que incluyera facultades de Humanidades, Ciencias Básicas, Ciencias de la Salud y Ciencias de la Ingeniería.

Se estudiaron aspectos asociados con interactividad y desplazamiento disciplinar estudiantil, prospectivas de desarrollo institucional y patrones cognitivos de identidad epistémica en diversos dominios disciplinares, en función de los campos comunicativos neurálgicos de la investigación. La Tabla 1 muestra las tres etapas principales.

El presente artículo solo reporta los resultados de la primera etapa de la investigación. Desde la base de estos resultados fueron proyectadas las dos instancias cualitativas descritas en la Tabla 1.

El protocolo metodológico se orientó al modelado cuantitativo de patrones de desplazamiento e interactividad disciplinar estudiantil en el contexto del currículo flexible de cada institución, mediante modelamiento estadístico asociado con las bases de datos del registro curricular de las tres universidades para un período de tres años. Se consideraron 264.270 operaciones de inscripción de cursos relacionadas con el currículo flexible u optativo de todas las carreras y los programas de pre y posgrado vigentes.

La oferta optativa global considerada en el estudio fue de 9.948 cursos, codificados cualitativamente

Tabla 1.

Diseño metodológico global de investigación

\begin{tabular}{|c|c|c|}
\hline $\begin{array}{l}\text { Primera etapa: modelado de procesos } \\
\text { de desplazamiento e interactividad } \\
\text { disciplinar }\end{array}$ & $\begin{array}{l}\text { Segunda etapa: análisis hermenéutico } \\
\text { documental }\end{array}$ & $\begin{array}{l}\text { Tercera etapa: análisis cognitivo y orga- } \\
\text { nizacional }\end{array}$ \\
\hline $\begin{array}{l}\text { Etapa destinada al análisis de patrones } \\
\text { estadísticos de recurrencia y correla- } \\
\text { ción en bases de datos y registro cu- } \\
\text { rricular, asociada con la descripción de } \\
\text { pautas de desplazamiento e interactivi- } \\
\text { dad disciplinar. }\end{array}$ & $\begin{array}{l}\text { Etapa destinada al análisis hermenéuti- } \\
\text { co documental de modelos curriculares, } \\
\text { prospectiva de desarrollo institucional y } \\
\text { programas de investigación. }\end{array}$ & $\begin{array}{l}\text { Etapa orientada al análisis de procesos } \\
\text { cognitivos de identidad intradisciplinar y } \\
\text { su proyección ecológica a redes de tra- } \\
\text { bajo inter y transdisciplinarias en investi- } \\
\text { gación y docencia universitaria. }\end{array}$ \\
\hline Fuentes de información & Fuentes de información & Fuentes de información \\
\hline $\begin{array}{l}\text { Bases de datos y registro curricular de } \\
\text { algunas universidades chilenas. }\end{array}$ & $\begin{array}{l}\text { Documentación institucional referida a } \\
\text { principios rectores de sus procesos for- } \\
\text { mativos e investigativos. }\end{array}$ & $\begin{array}{l}\text { Observación participante, entrevistas en } \\
\text { profundidad y grupos de discusión con } \\
\text { actores clave y pertenencia disciplinar. }\end{array}$ \\
\hline $\begin{array}{l}\text { Codificación de cursos y carreras me- } \\
\text { diante criterios OCDE. }\end{array}$ & $\begin{array}{l}\text { Interpretación y codificación cualitativa } \\
\text { inductiva-deductiva. }\end{array}$ & \multirow{2}{*}{$\begin{array}{l}\text { Codificación cualitativa inductiva-deduc- } \\
\text { tiva, análisis de frecuencia y asociación } \\
\text { de códigos y modelado de patrones cog- } \\
\text { nitivos locales y globales. }\end{array}$} \\
\hline Estadística descriptiva y correlacional. & & \\
\hline
\end{tabular}

Fuente: elaboración propia

3 En función de los criterios de confidencialidad que oficiaron de base a la investigación, solo se señalan aspectos generales del perfil institucional de las tres universidades incluidas en el estudio.
4 Comisión Nacional de Acreditación. En Chile es la instancia encargada de la acreditación de carreras e instituciones de educación superior. 
en función de la tipología disciplinar (OCDE, 2008; 2009). La clasificación definió seis dominios: Ciencias Naturales, Ingeniería y Tecnología, Medicina y Ciencias de la Salud, Ciencias de la Agricultura, Ciencias Sociales, Humanidades y Otros. Los criterios de codificación para cada curso consideraron los siguientes ítems en orden jerárquico: a) características temáticas del curso; b) unidad académica que dicta el curso; c) perfil estudiantil adscrito al curso, y d) consideraciones adicionales (programa de curso, referencias del docente y contexto de desarrollo). Para la codificación de la pertenencia disciplinar estudiantil se tomaron en cuenta los campos de las facultades a las cuales se adscriben las carreras.

Como patrón de desplazamiento disciplinar fue definido todo proceso de migración o movilidad de estudiantes entre áreas disciplinares diversas. Se utilizó como indicador el cruce entre la pertenencia disciplinar de cada estudiante (programa de estudios en el que está agenciado) y la pertenencia disciplinar de los cursos inscritos en el contexto del currículo flexible de cada institución.

Asimismo, se definió como patrón de interacción disciplinar al grado de diversidad de estudiantes partícipes de un mismo ambiente de aprendizaje formal (cursos del currículo flexible inscritos en los registros académicos). Se empleó como indicador el número de áreas disciplinares (representadas por los estudiantes) inscritos en cada curso específico 5 .

Luego de la codificación de cursos, se analizaron las frecuencias de las inscripciones de los estudiantes según el dominio disciplinar de pertenencia (patrón de desplazamiento disciplinar) y las frecuencias del número de estudiantes de distintos dominios disciplinares por cada asignatura analizada (patrón de interacción disciplinar).

\section{Resultados}

\subsection{Caracterización general de la oferta curricular flexible}

En los casos 1 y 2, la oferta curricular flexible presentó un número significativo de cursos en función de la población estudiantil, una equitativa presencia de los diversos dominios disciplinares en cuanto a contenidos y perfil docente y una sostenida oferta durante los tres años. El caso 3 mostró una constante oferta curricular vinculada al currículo optativo; no obstante, esta se centró en cursos pertenecientes solo al dominio de las humanidades.

En términos generales, estos antecedentes configuran condiciones iniciales relativamente favorables para la emergencia de enfoques formativos inter/transdisciplinarios en los procesos de la mayoría de las carreras profesionales impartidas en estas tres universidades, si se consideran los campos disciplinares básicos de cada una de ellas y las posibilidades de interacción entre docentes y estudiantes.

\subsection{Modelamiento de procesos de desplazamiento disciplinar}

Los resultados evidencian restringidos procesos de desplazamiento e interactividad estudiantil y docente entre los diversos dominios disciplinares, con prevalencia de dinámicas de autorreferencia y aislamiento disciplinar en términos formativos.

La Figura 1 muestra la distribución de estudiantes según su dominio disciplinar y los cursos del currículo flexible, codificados según tipología OCDE y los criterios ya señalados. Como muestran las gráficas, es posible observar que los estudiantes agenciados a cada dominio disciplinar tienden, en términos de regularidades globales, a inscribir y participar de cursos del currículo flexible dentro de las cotas de su mismo dominio disciplinar.

5 De manera análoga, estudios que evalúan interacción disciplinar entre grupos de investigación han utilizado la participación conjunta de diversos autores en publicaciones o proyectos específicos como indicador de interacción o colaboración; ejemplo de ello es la investigación de Cárdenas y otros autores (2012). 
Figura 1.

Desplazamiento disciplinar desglosado por área disciplinar de estudiantes para cada caso
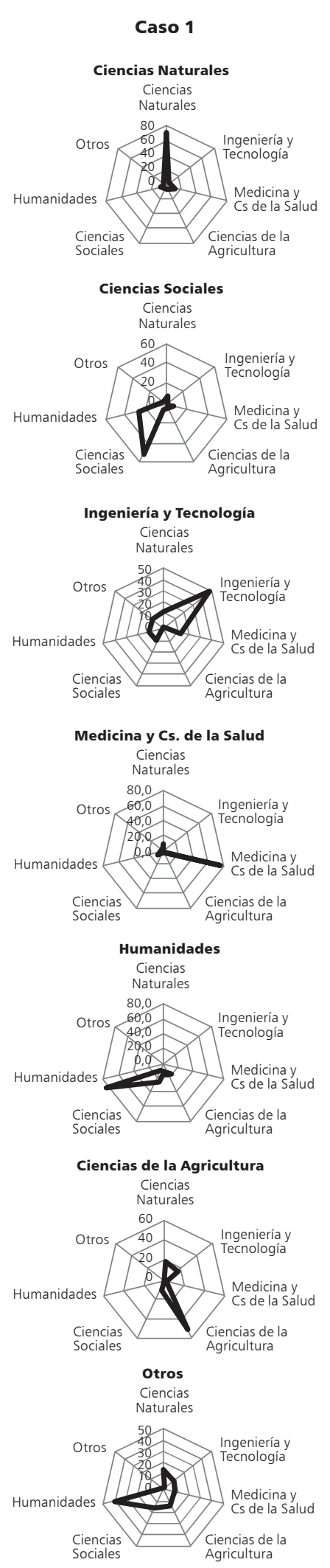

Fuente: elaboración propia
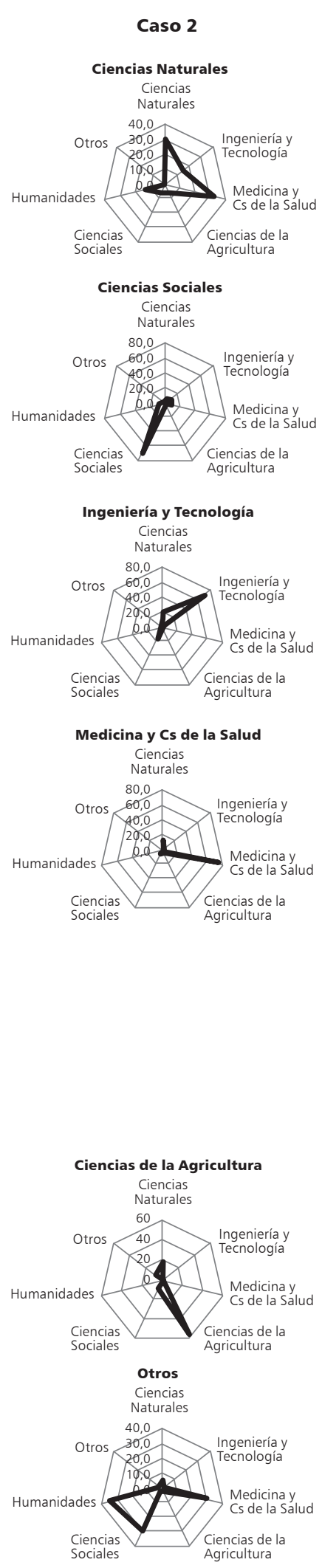
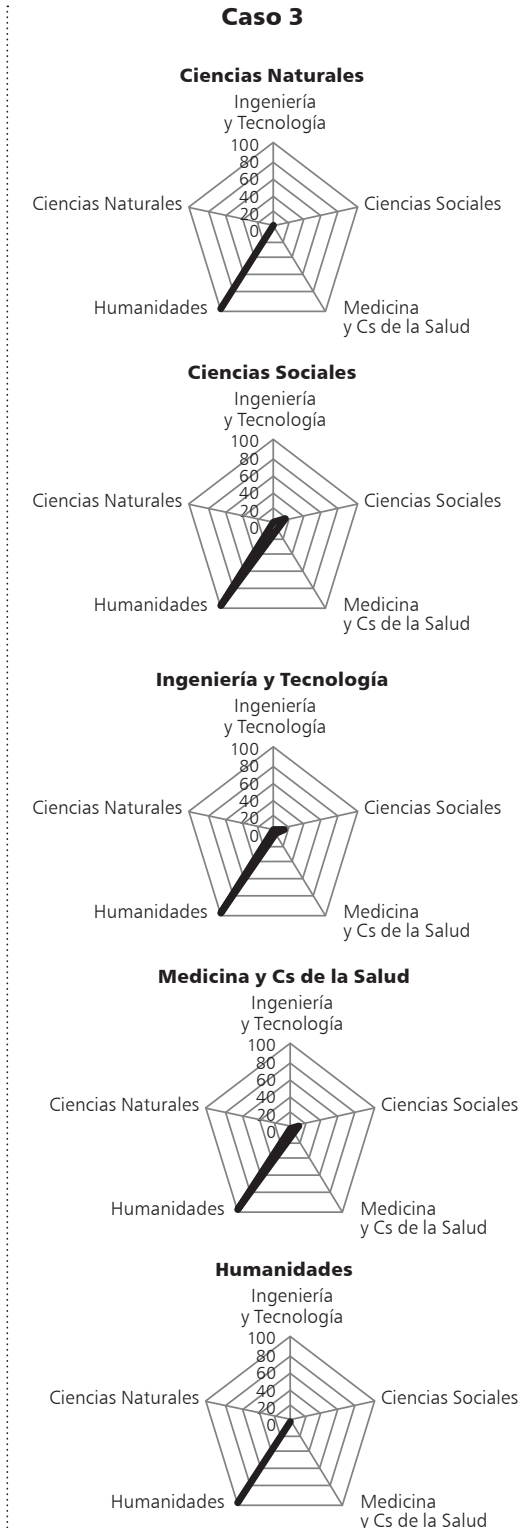

Medicina
y Cs de la Salud 
Al neutralizar las inscripciones de cursos dentro del mismo dominio disciplinar, la Figura 2 representa el desplazamiento estudiantil según su pertenencia disciplinar de base.

Figura 2.

Gráficas desglosadas por cada caso

\section{Caso 1}

Humanidades

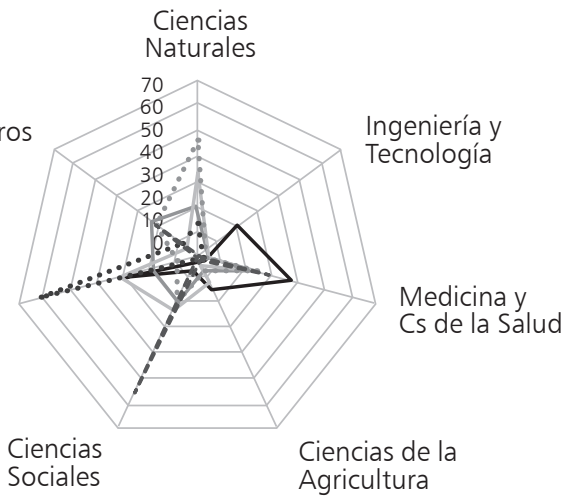

Caso 2

Caso 3

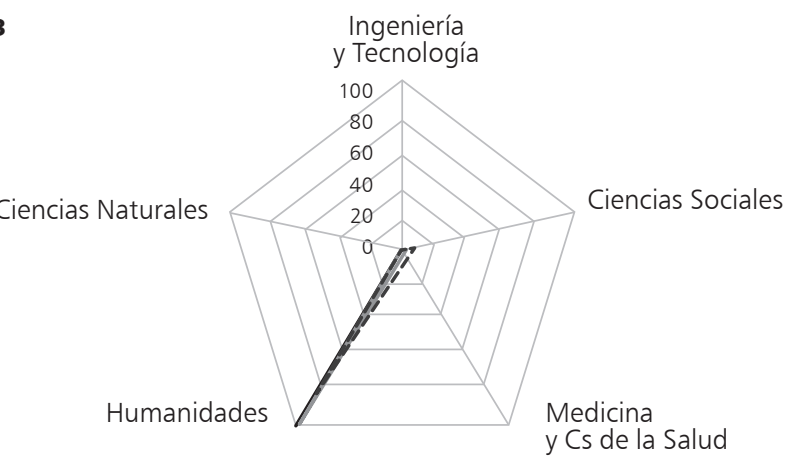

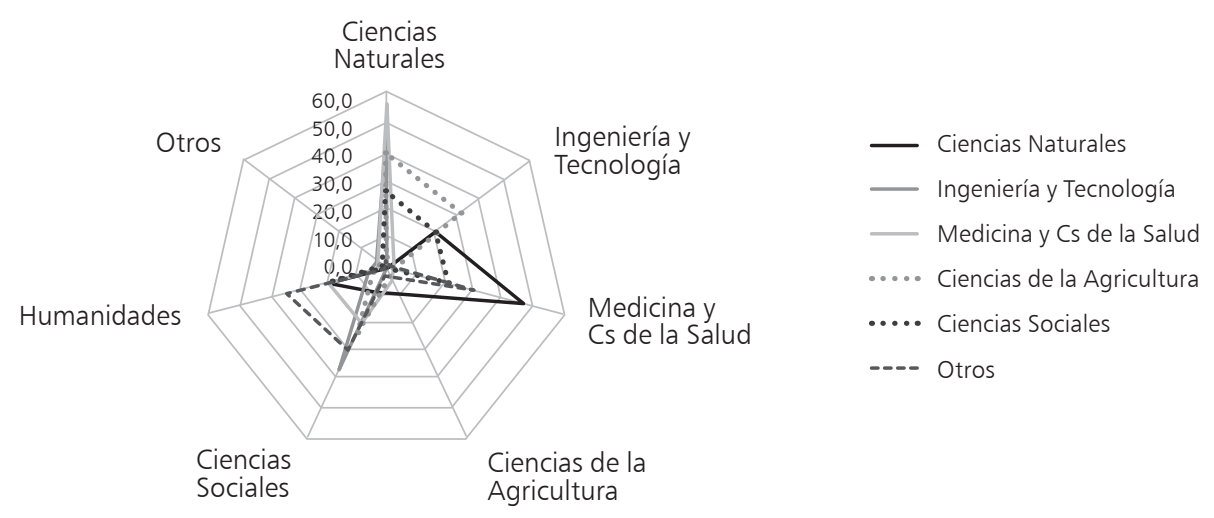

Ingeniería y Tecnología

Medicina y Cs de la Salud

— Ciencias Agrícolas

..... Ciencias Sociales

..... Humanidades

---- Otros

Fuente: elaboración propia

Incluye todos los procesos de inscripción de cursos del currículo flexible de pregrado y posgrado para cada institución. Se eliminó el efecto de autorreferencia disciplinar, para dar mayor sensibilidad a los efectos de desplazamiento disciplinar remanentes. 
Sin desconocer el componente de clausura disciplinar evidenciado, es importante señalar que los cursos asociados con el campo de las humanidades y ciencias sociales tienden a comportarse como convocantes de otros dominios disciplinares; no obstante, los estudiantes de ambas áreas tienen baja migración hacia otros campos disciplinares y profesionales. Como antecedente adicional, en los tres casos no hubo diferencias significativas en cuanto a desplazamiento disciplinar de estudiantes de pregrado y posgrado.

\subsection{Modelamiento de procesos de interactividad disciplinar}

El componente de interactividad es fundamental: desde la base del supuesto de que una mayor presencia de estudiantes de diversas áreas disciplinares dentro de un mismo curso propicia condiciones iniciales favorables para la construcción de matrices comunicativas multirreferenciales y aprendizajes interdisciplinarios.

Figura 3.

Distribución de cursos por dominio disciplinar, según grados de diversidad de pertenencia disciplinar de estudiantes inscritos

Caso 1

$$
\begin{array}{r}
\text { Otros } \\
\text { Humanidades } \\
\text { Ciencias Sociales } \\
\text { Ciencias de la Agricultura } \\
\text { Medicina y Cs de la Salud } \\
\text { Ingeniería y Tecnología } \\
\text { Ciencias Naturales }
\end{array}
$$

Solo dominio disciplinar correspondiente

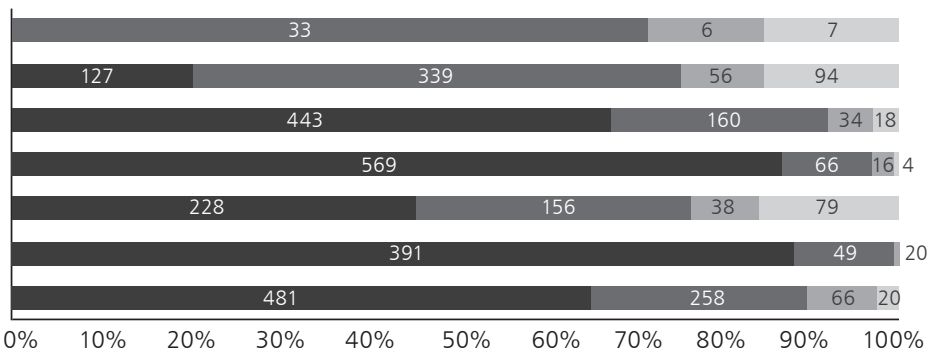

1 dominio disciplinar externo

2 dominios disciplinares externos 3 o más dominios disciplinares externos

Caso 2

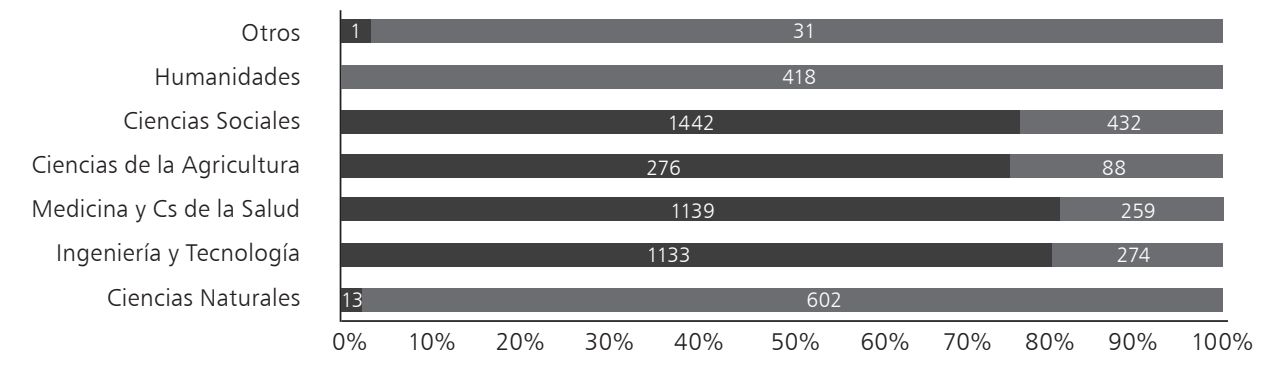

- Solo dominio disciplinar correspondiente 1 dominio disciplinar externo

Caso 3

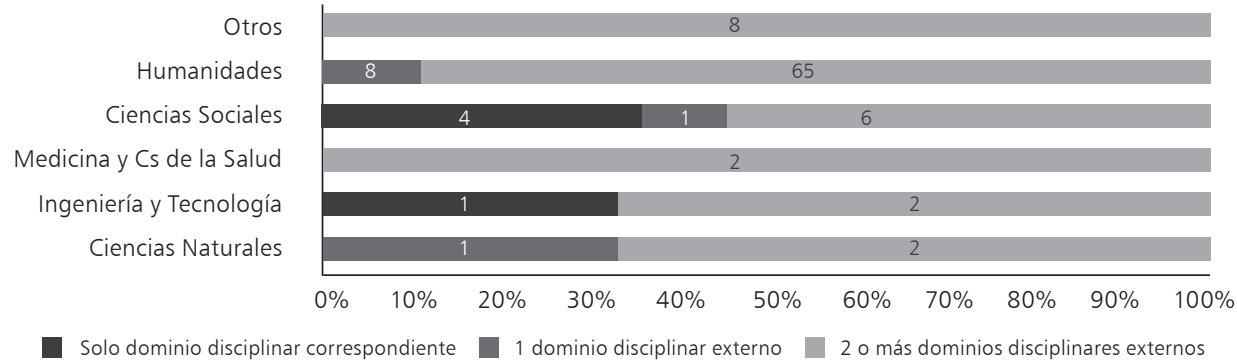

Fuente: elaboración propia 
Para interpretar los resultados expresados en la Figura 3, es necesario precisar que la naturaleza de la oferta del currículo flexible en las tres universidades presenta especificidades estructurales, lo que, más allá del desplazamiento disciplinar estudiantil, tiene implicaciones en su interactividad y pluralidad de perspectivas disciplinares presentes en cada curso.

En el caso 1, el currículo flexible (optativos y facultativos) está abierto a todas las carreras de la universidad, lo que permite desplazamiento e interactividad disciplinar. En el caso 2, la oferta flexible está dirigida a carreras específicas (el currículo flexible se dicta para cada carrera), lo que también fomenta el desplazamiento disciplinar de los estudiantes y su formación en otros contextos disciplinares y profesionales, pero no así la interactividad entre diversas disciplinas. Por último, en el caso 3, un gran porcentaje de la oferta del currículo flexible proviene de un solo dominio disciplinar: las humanidades. Esto explica la alta convergencia de los diversos perfiles disciplinares de estudiantes en este tipo de cursos.

Es necesario señalar que en el caso 2 se observó un relativo desplazamiento disciplinar asociado con una baja interactividad estudiantil intracursos, producto de lo descrito en el párrafo anterior. En el caso 3, los estudiantes tienen un desplazamiento disciplinar muy focalizado hacia una sola área (debido a las características de la oferta curricular flexible), pero alta interactividad entre disciplinas en cada curso.

\section{Discusiones y conclusiones}

El objetivo de la investigación fue levantar información que permitiera evaluar, de manera preliminar, las regularidades cuantitativas referentes a los procesos de desplazamiento e interactividad disciplinar entre estudiantes de diversas carreras de acuerdo con la totalidad de estudiantes de pregrado y posgrado de cada institución. Se evaluó la flexibilidad operativa del currículo optativo, entendida como condicionante inicial para la emergencia y configuración de esquemas inter/transdisciplinares en los procesos formativos de estas instituciones. Pese al carácter central de la noción de flexibilidad curricular en este trabajo, es necesario reconocer que solo representa una aproximación parcial y operativa y que de ningún modo circunscribe todos los recursos epistemológicos y metodológicos de desarrollo de la inter/transdisciplinariedad en espacios universitarios.

En este contexto, la caracterización del currículo flexible permitió describir un significativo número de cursos en función de la población estudiantil, equitativa presencia de los diversos dominios disciplinares en cuanto a contenidos y perfil docente y una sostenida oferta durante el período. No obstante, la operatividad del currículo flexible es aún insuficiente y su presencia es poco significativa para la gran mayoría de los estudiantes, como se ve en su comportamiento en términos de inscripción de cursos.

En términos globales, los resultados muestran que el desplazamiento disciplinar de los estudiantes de todos los dominios disciplinares tiende a la endogamia y autorreferencia, lo que condiciona significativamente la operatividad un currículo flexible e interdisciplinario. Esto puede deberse a que los estudiantes ven la oferta optativa y flexible como un recurso de profundización y de mayor especialización, antes que como posibilidad de transitar y complementar su formación profesional particular con otras perspectivas disciplinares en un contexto formativo más global. Esto impide que profesionales de diversas disciplinas operen desde una síntesis interpretativa más compleja de la sociedad, lo que en definitiva da sentido y pertinencia a su especificidad disciplinar o profesional. 
Desde una óptica complementaria, es preciso estudiar el perfil de gestión académica de las direcciones de carrera (o equivalentes en cada institución), respecto a evaluar cómo se favorece o condiciona la formación inter/transdisciplinaria de sus estudiantes en el contexto específico de la operatividad del currículo flexible. Esto, debido a que, bajo paradigmas endogámicos y de especificidad disciplinar, tienden a obstaculizar el desplazamiento disciplinar de sus estudiantes.

Resultados como los reportados en este artículo no solo alertan sobre las formas de organización y comunicación entre los diversos sistemas de conocimiento o campos disciplinares de las universidades consideradas en la investigación, sino que además visibilizan restrictivas formas de vivir la universidad. Estos patrones de aislamiento y autorreferencia entre los diversos sistemas de conocimiento que conforman la universidad-sociedad contemporánea y el predominio de dinámicas de aprendizaje paralelas o divergentes, evidencian condicionantes importantes para la construcción de aprendizajes más complejos y multirreferenciales.

En cuanto a las limitaciones del estudio, hay que precisar que esta etapa de investigación solo mostró patrones globales de desplazamiento e interactividad disciplinar desde una óptica cuantitativa; por ello, no permite inferir las dinámicas internas, las formas de aprendizaje potenciadas en cada curso y la naturaleza cualitativa e interpretativa de la relación comunicativa entre disciplinas. Esto último, en convergencia con los diversos antecedentes teóricos y metodológicos ya discutidos, abre nuevas perspectivas de investigación orientadas a profundizar, tensionar y triangular, desde cánones metodológicos cualitativos complementarios, los principios de identidad disciplinar (en referencia apertura y clausura a otros sistemas de conocimiento) y los procesos interpretativos de los diversos actores en torno a los grados de pertinencia y legitimidad de la inter/transdisciplinariedad en los procesos de generación y comunicación de conocimiento en las universidades contemporáneas.

\section{Sobre los autores}

Iván Oliva-Figueroa es licenciado en Biología, doctor en Ciencias de la Educación. Profesor de Biología, académico del Instituto de Filosofía y Estudios Educacionales, de la Facultad de Filosofía y Humanidades de la Universidad Austral de Chile. Es director del Programa de Maestría en Educación, Mención Política y Gestión Educativas. La principal línea de investigación está asociada con la comprensión de condicionantes y posibilidades organizacionales referidas al desarrollo de esquemas de inter/transdisciplinariedad en educación terciaria.
Tomas Koch-Ewertz es licenciado en Sociología, magíster en Educación, Mención Política y Gestión Educativas, académico del Departamento de Sociología, de la Facultad de Ciencias Sociales, de la Universidad de Playa Ancha. La principal línea de investigación se refiere a sociología de la educación, sociología del conocimiento y metodología de la investigación en Ciencias Sociales.

John Quintero-Tapia es licenciado en Educación Básica con énfasis en Educación Física, Recreación y Deportes, magíster en Educación, Mención Política y Gestión Educativas. Es secretario académico del Programa de Pedagogía Media en Educación Física, de la Universidad San Sebastián, sede Valdivia. La principal línea de investigación se asocia con procesos de formación de profesores y formación en investigación.

\section{Referencias}

Austin, W., Park, C., \& Goble, E. (2008). From Interdisciplinary to Transdisciplinary Research: A Case Study. Qualitative Health Research, 18 (4) 557-564.

Berman, M. (1981). El reencantamiento del mundo. Santiago: Cuatro vientos.

Bunge, M. (1991). Sociología de la ciencia. Buenos Aires: Sudamericana.

Bunge, M. (2004). Emergencia y convergencia. Novedad cualitativa y unidad del conocimiento. BarceIona: Gedisa.

Cáceres, C. (2007). Planeación estratégica en universidades del Consejo de Rectores: evidencias del periodo 2000-2005. Calidad de la Educación, 27 (2), 108-150.

Cáceres, C., \& Cristi, O. (2003). Cooperar desde la diversidad. Calidad de la Educación, 19 (2). Recuperado de http://www.cned.cl/public/secciones/ seccionrevistacalidad/doc/37/cse_articulo189.pdf

Capra, F. (2002). Las conexiones ocultas. Barcelona: Anagrama.

Cárdenas, J., Olivares, G., Alfaro, R., Cabrera, F; Gibert, J; Goya, Samaniego, H. \& Palacios, A. (2012). Estudio de la colaboración científica de la región de Valparaíso de Chile desde la perspectiva de las redes complejas. Recuperado de http://www. senado.cl/prontus_senado/site/artic/20120518/ asocfile/20120518144329/colaboracion_cientifica_iscv_jua_pablo_cardenas.pdf

Centro Micro Datos (2008). Informe final: estudio sobre causas de la deserción universitaria. Santiago: Universidad de Chile.

Díaz, N. (2010). Transdisciplina y educación terciaria en el sur de Chile. Estudio paradigmatológico y análisis hermenéutico documental desde la teoría del pensamiento complejo de Edgar Morin. (Tesis de pregrado). Universidad Austral de Chile, Valdivia, Chile. 
Feyerabend, P. (1975). Tratado contra el método. Barcelona: Folio.

Flórez, A., \& Millán, C. (ed.) (2002). Desafíos de la transdisciplinariedad. Bogotá: Pontificia Universidad Javeriana.

Gaete, M., \& Morales, R. (2011). Articulación del sistema de educación superior en Chile. Posibilidades, tensiones y desafíos. Calidad de la Educación, (35), 51-89.

Gherab, K. (2009). Interdisciplinariedad y redes epistemológicas de la ciencia en internet. Arbor Ciencia, pensamiento y cultura, CLXXXV (737), 611-622.

González, E. (2011). La nueva juventud y el proceso de transición entre la educación media y superior. En El proceso de transición entre educación media y superior. Experiencias universitarias. Santiago: Cinda.

Greckhamer, T., Kro-Ljungberg, M., Cilesiz, S., \& Hayes, S. (2008). Demystifying Interdisciplinary Qualitative. Research Qualitative Inquiry, 14 (2) 307-331.

Hirsch Hadorn, G., Hoffmann-Riem, H., Biber-Klemm, S., GrossenbacherMansuy, W., Joye, D., Pohl, C. et al. (eds.) (2008). Handbook of Transdisciplinary Research. Berna: Springer Science.

Jeffrey, P. (2003). Smoothing the Waters: Observations on the Process of Cross-Disciplinary Research Collaboration. Social Studies of Science, (33), 539-562.

Kelly, K. (1995). Out of Control: The Rise of Neo-Biological Civilization. Menlo Park: Addison-Wesley.

Kuhn, T. (1962). The Structure of Scientific Revolutions. Chicago: University of Chicago Press.

Lakatos, I., \& Musgrave, A. (1970). Criticism and the Growth of Knowledge. Cambridge: Cambridge University Press.

Leavy, P. (2011). Essentials of Transdisciplinarity Research. Walnut Creek: Left Coast Press.

Lenoir, Y., Hasni, A., \& Larose, F. (2007). L'interdisciplinarité et la formation à I'enseignement: analyse de résultats de deuxrecherches. Pensamiento Educativo, 4 (2), 255-276.

Miñana, C. (2002). Interdisciplinariedad y currículo. Un estado del arte. En C. Miñana (ed.), Memorias Seminario internacional sobre interdisciplinariedad y currículo. Construcción de proyectos Escuela-Universidad. Bogotá: Universidad Nacional de Colombia, Programa RED.

Motta, R. (2000). Complejidad, educación y transdisciplinariedad. Signos Universitarios, $X X(37), 69-92$

Morin, E. (1992). El método. Las ideas. Madrid: Cátedra.

Morin, E. (1998). El método: El conocimiento del conocimiento. Madrid: Cátedra.

Morin, E. (1999). La cabeza bien puesta: Repensar la reforma Bà Reformar el pensamiento. Buenos Aires: Nueva Visión.

Morin, E. (2006). El método. Ética. Madrid: Cátedra.

Morin, E. (2008). On Complexity. Nueva Jersey: Hampton Press.

Nemeth-Baumgartner, A. (1993). Macrometanoia. Un nuevo orden, una nueva civilización. Santiago: Sudamericana.

Nicolescu, B. (1996). La transdisciplinariedad. Manifiesto. (Núñez \& Gérard, trad.). París. Ediciones Du Rocher.

Organización para la Cooperación y el Desarrollo Económicos [OCDE]. (2008). Tertiary Education for the Knowledge Society. Thematic Review of Tertiary Education, 1-3.

OCDE. (2009). Educación Superior en Chile. Santiago: Ministerio de Educación.

Consejo Asesor Presidencial para la Educación Superior. (2008). Los desafíos de la educación superior chilena. Santiago: Autor. 
Oliva, I. (2008). Conocimiento, universidad y complejidad: bosquejos epistémicos y metodológicos para una vinculación transdisciplinaria. Estudios Pedagógicos, 34 (2), 227-243.

Oliva, I. (2010). Breve cartografía a una disonancia epistémica: educación, complejidad y reforma. Polis, 9 (25), 321-335.

Parodi, G. (2008). Géneros académicos y géneros profesionales: accesos discursivos para saber y hacer. Valparaíso: Ediciones Universitarias de Valparaíso.

Piaget, J. (1966). Problémes généraux de la recherche insterdisciplinare et mécanismes communs. París: Unesco.

Reynoso, C. (2011). Redes sociales y complejidad. Modelos interdisciplinarios en la gestión sostenible de la sociedad y la cultura. Buenos Aires: sb.

Reynoso, C. (2009). Modelos o metáforas. Crítica del paradigma de la complejidad de Edgar Morin. Buenos Aires: sb.

Rosnay, J. (1977). El macroscopio: hacia una visión global. Madrid: AC.

Salazar, J. (2003). Convergencia e institucionalidad en la educación superior. Calidad de la educación, 19 (2), 37-50. Recuperado de http:// www.cned.cl/public/secciones/seccionpublicaciones/doc/37/cse_articulo190.pdf

Silva, D. (2011). Educación terciaria y transdisciplina: una aproximación a patrones de movimiento disciplinar estudiantil. (Tesis de maestría inédita). Universidad Austral de Chile, Valdivia.

Soler, A. (2011). Conocimiento, complejidad y transdisciplina: aproximación hermenéutica a las prospectivas de desarrollo universitario en el sur de Chile. (Tesis de maestría inédita). Universidad Austral de Chile, Valdivia.

Villaroel, P., \& Firmani, C. (2002). Necesidad del enfoque transdisciplinario en la investigación ambiental académica. IV Encuentro Científico del Medio Ambiente. Antofagasta, CIPMA. 